\title{
DECOMPOSITION OF PEANO DERIVATIVES
}

\author{
HAJRUDIN FEJZIĆ
}

(Communicated by Andrew Bruckner)

\begin{abstract}
Let $\Delta^{\prime}$ be the class of all derivatives, and let $\left[\Delta^{\prime}\right]$ be the vector space generated by $\Delta^{\prime}$ and O'Malley's class $B_{1}^{*}$. In [1] it is shown that every function in $\left[\Delta^{\prime}\right]$ is of the form $g^{\prime}+h k^{\prime}$, where $g, h$, and $k$ are differentiable, and that $f \in\left[\Delta^{\prime}\right]$ if and only if there is a sequence of derivatives $v_{n}$ and closed sets $A_{n}$ such that $\bigcup_{n=1}^{\infty} A_{n}=\mathbf{R}$ and $f=v_{n}$ on $A_{n}$. The sequence of sets $A_{n}$ together with the corresponding functions $v_{n}$ is called a decomposition of $f$. In this paper we show that every Peano derivative belongs to $\left[\Delta^{\prime}\right]$. Also we show that for Peano derivatives the sets $A_{n}$ can be chosen to be perfect.
\end{abstract}

\section{INTRODUCTION}

Let $C$ be the family of all continuous functions on $\mathbf{R}, \Delta$ the family of all differentiable functions on $\mathbf{R}$ and $\Delta^{\prime}$ the family of all derivatives on $\mathbf{R}$. If $\Gamma$ is a family of functions defined on $\mathbf{R}$, then by $[\Gamma]$ we denote the family of all functions $f$ on $\mathbf{R}$ with the following property: for every $n=1,2, \ldots$ there exist $v_{n} \in \Gamma$ and closed sets $A_{n}$ such that $\bigcup_{n=1}^{\infty} A_{n}=\mathbf{R}$ and $f=v_{n}$ on $A_{n}$. In [1, Theorem 2] it is shown that the following four conditions are equivalent:

(i) There are $g, h$, and $k$ in $\Delta$ such that $h^{\prime}, k^{\prime} \in[C]$ and $f=g^{\prime}+h k^{\prime}$.

(ii) There is a $\varphi \in \Delta^{\prime}$ and $\psi \in[C]$ such that $f=\varphi+\psi$.

(iii) $f \in\left[\Delta^{\prime}\right]$.

(iv) There is a dense open set $T$ and a function $k \in \Delta$ such that $f$ is a derivative on $T$ and $f=k^{\prime}$ on $\mathbf{R} \backslash T$.

Statement (ii) implies that $\left[\Delta^{\prime}\right]$ is a vector space generated by $\Delta^{\prime}$ and $[C]$. In $[1$, Theorem 3$]$ it is shown that each approximate derivative, each approximately continuous function, and each function in $B_{1}^{*}=[C]$ belongs to the class $\left[\Delta^{\prime}\right]$. In [5] O'Malley showed that for approximate derivatives sets $A_{n}$ from the definition of $\left[\Delta^{\prime}\right]$ can be chosen to be perfect. A question raised in [1] is: "Does every Peano derivative belong to $\left[\Delta^{\prime}\right]$ ?"

The main goal of this paper is to show that a $k$ th Peano derivative is in $\left[\Delta^{\prime}\right]$ and that the sets $A_{n}$ from the definition of $\left[\Delta^{\prime}\right]$ can be chosen to be perfect. We will prove even more, namely, that a $k$ th Peano derivative is a composite derivative of the $(k-1)$ th Peano derivative. An immediate consequence of this result is that a $k$ th Peano derivative is an approximate derivative of the

Received by the editors March 4, 1992.

1991 Mathematics Subject Classification. Primary 26A24. 
$(k-1)$ th Peano derivative almost everywhere. This result was first proved by Zygmund and Marcinkiewicz (see [12, p. 77]).

\section{Preliminaries}

In this section we will recall the definition of Peano derivatives and some known properties of Peano derivatives.

Definition 1. Let $f$ be a continuous function defined on $\mathbf{R}$. We say that $f$ has $k$ th Peano derivative at some point $x$, if there are real numbers $f_{1}, f_{2}, \ldots, f_{k}$ such that

(1) $f(x+h)=f(x)+h f_{1}+\cdots+h^{k} \frac{f_{k}}{k !}+h^{k} \varepsilon_{k}(h)$ where $\lim _{h \rightarrow 0} \varepsilon_{k}(h)=0$.

The number $f_{k}$ is called the $k$ th Peano derivative of $f$ at $x$, and since it depends only on a function $f$ and a point $x$, it will be convenient to denote it by $f_{k}(x)$. Similarly the continuous function $\varepsilon_{k}(h)$ depends on $x$, so we may denote it by $\varepsilon_{k}(x, h)$. Also, it will be convenient to denote $f(x)$ by $f_{0}(x)$. With this notation (1) becomes $f(x+h)=\sum_{j=0}^{k} h^{j} \frac{f_{j}(x)}{j !}+h^{k} \varepsilon_{k}(x, h)$. From Definition 1 it is easy to see that if the $k$ th Peano derivative exists, so does the $n$ th, for $1 \leq n<k$.

It is known that the $k$ th Peano derivative is Baire 1, Darboux, and has Denjoy property. For these, and some other properties of Peano derivatives, see [3, 4, $6,8-12]$.

\section{Formula}

In this section we will derive a formula that is the crux of the proof of Theorem 2.

Theorem 1. Let $f$ be a continuous function on $\mathbf{R}$, and let $x \neq x_{1}$ and $t \neq 0$ be points such that $f_{k}(x)$ and $f_{k}\left(x_{1}\right)$ exist. Then the following formula holds:

$$
\begin{aligned}
& \frac{f_{k-1}\left(x_{1}\right)-f_{k-1}(x)}{x_{1}-x}-f_{k}(x) \\
& =\frac{t}{x_{1}-x} \frac{k-1}{2} f_{k}(x) \\
& \quad+\sum_{j=0}^{k-1}(-1)^{k-1-j}\left(\begin{array}{c}
k-1 \\
j
\end{array}\right) \frac{\left(x_{1}-x+j t\right)^{k}}{t^{k-1}\left(x_{1}-x\right)} \varepsilon_{k}\left(x, x_{1}-x+j t\right) \\
& \quad-\frac{t}{x_{1}-x}\left\{\frac{k-1}{2} f_{k}\left(x_{1}\right)+\sum_{j=0}^{k-1}(-1)^{k-1-j}\left(\begin{array}{c}
k-1 \\
j
\end{array}\right) j^{k} \varepsilon_{k}\left(x_{1}, j t\right)\right\} .
\end{aligned}
$$

To prove this formula we need some lemmas.

Lemma 1. For $m \in \mathbf{N}$ we have

$$
\sum_{j=0}^{m}(-1)^{m-j}\left(\begin{array}{c}
m \\
j
\end{array}\right) j^{i}= \begin{cases}0 & \text { if } i=0,1, \ldots, m-1 \\
m ! & \text { if } i=m \\
\frac{m}{2}(m+1) ! & \text { if } i=m+1\end{cases}
$$


Proof. The case $0 \leq i \leq m$ is a well-known exercise in mathematical induction. So let us consider only the case $i=m+1$. Let

$$
a(m)=\sum_{j=0}^{m}(-1)^{m-j}\left(\begin{array}{c}
m \\
j
\end{array}\right) j^{m+1} .
$$

Then we have the following recursive formula: $a(m)=m a(m-1)+m m$ ! , and since $a(1)=1$, we have $a(m)=\frac{m}{2}(m+1)$ !

Definition 2. Let $f$ be a function defined on $\mathbf{R}$. Then for any two points $x_{1}$ and $t$ let

$$
\Delta_{k-1}=\sum_{j=0}^{k-1}(-1)^{k-1-j}\left(\begin{array}{c}
k-1 \\
j
\end{array}\right) f\left(x_{1}+j t\right)
$$

Lemma 2. Let $f$ be a function defined on $\mathbf{R}$ having a kth Peano derivative at some point $x_{1}$. Then for any $t$ the following holds:

$$
\Delta_{k-1}=t^{k-1} f_{k-1}\left(x_{1}\right)+t^{k} \frac{k-1}{2} f_{k}\left(x_{1}\right)+t^{k} \sum_{j=0}^{k-1}(-1)^{k-1-j}\left(\begin{array}{c}
k-1 \\
j
\end{array}\right) j^{k} \varepsilon_{k}\left(x_{1}, j t\right) .
$$

Proof.

$$
\begin{aligned}
\Delta_{k-1}= & \sum_{j=0}^{k-1}(-1)^{k-1-j}\left(\begin{array}{c}
k-1 \\
j
\end{array}\right)\left(\sum_{l=0}^{k}(j t)^{l} \frac{f_{l}\left(x_{1}\right)}{l !}+(j t)^{k} \varepsilon_{k}\left(x_{1}, j t\right)\right) \\
= & \sum_{l=0}^{k}\left(\sum_{j=0}^{k-1}(-1)^{k-1-j}\left(\begin{array}{c}
k-1 \\
j
\end{array}\right) j^{l}\right) t^{l} \frac{f_{l}\left(x_{1}\right)}{l !} \\
& +t^{k} \sum_{j=0}^{k-1}(-1)^{k-1-j}\left(\begin{array}{c}
k-1 \\
j
\end{array}\right) j^{k} \varepsilon_{k}\left(x_{1}, j t\right) .
\end{aligned}
$$

The rest follows from Lemma 1.

Lemma 3. Let $f$ be a function on $\mathbf{R}$, and let $x \neq x_{1}$ be a point such that $f_{k}(x)$ exists. Then

$$
\begin{aligned}
\Delta_{k-1}= & t^{k-1} f_{k-1}(x)+t^{k-1}\left(x_{1}-x\right) f_{k}(x)+t^{k} \frac{k-1}{2} f_{k}(x) \\
& +\sum_{j=0}^{k-1}(-1)^{k-1-j}\left(\begin{array}{c}
k-1 \\
j
\end{array}\right)\left(x_{1}-x+j t\right)^{k} \varepsilon_{k}\left(x, x_{1}-x+j t\right) .
\end{aligned}
$$


Proof.

$$
\begin{aligned}
\Delta_{k-1}= & \sum_{j=0}^{k-1}(-1)^{k-1-j}\left(\begin{array}{c}
k-1 \\
j
\end{array}\right) \\
& \cdot\left(\sum_{l=0}^{k}\left(x_{1}-x+j t\right)^{l} \frac{f_{l}(x)}{l !}+\left(x_{1}-x+j t\right)^{k} \varepsilon_{k}\left(x, x_{1}-x+j t\right)\right) \\
= & \sum_{j=0}^{k-1}(-1)^{k-1-j}\left(\begin{array}{c}
k-1 \\
j
\end{array}\right) \sum_{l=0}^{k}\left(x_{1}-x+j t\right)^{l} \frac{f_{l}(x)}{l !} \\
& +\sum_{j=0}^{k-1}(-1)^{k-1-j}\left(\begin{array}{c}
k-1 \\
j
\end{array}\right)\left(x_{1}-x+j t\right)^{k} \varepsilon_{k}\left(x, x_{1}-x+j t\right) .
\end{aligned}
$$

Since

$$
\begin{aligned}
& \sum_{j=0}^{k-1}(-1)^{k-1-j}\left(\begin{array}{c}
k-1 \\
j
\end{array}\right) \sum_{l=0}^{k}\left(x_{1}-x+j t\right)^{l} \frac{f_{l}(x)}{l !} \\
& =\sum_{j=0}^{k-1}(-1)^{k-1-j}\left(\begin{array}{c}
k-1 \\
j
\end{array}\right) \sum_{l=0}^{k} \sum_{i=0}^{l}\left(\begin{array}{l}
l \\
i
\end{array}\right)\left(x_{1}-x\right)^{l-i}(j t)^{i} \frac{f_{l}(x)}{l !} \\
& =\sum_{l=0}^{k} \sum_{i=0}^{l}\left(\begin{array}{l}
l \\
i
\end{array}\right)\left(x_{1}-x\right)^{l-i} t^{i}\left(\sum_{j=0}^{k-1}(-1)^{k-1-j}\left(\begin{array}{c}
k-1 \\
j
\end{array}\right) j^{i}\right) \frac{f_{l}(x)}{l !},
\end{aligned}
$$

by Lemma 1 it is equal to

$$
\sum_{l=k-1}^{k} \sum_{i=k-1}^{l}\left(\begin{array}{l}
l \\
i
\end{array}\right)\left(x_{1}-x\right)^{l-i} t^{i}\left(\sum_{j=0}^{k-1}(-1)^{k-1-j}\left(\begin{array}{c}
k-1 \\
j
\end{array}\right) j^{i}\right) \frac{f_{l}(x)}{l !} .
$$

Applying Lemma 1 once more it is equal to

$$
t^{k-1} f_{k-1}(x)+t^{k-1}\left(x_{1}-x\right) f_{k}(x)+t^{k} \frac{k-1}{2} f_{k}(x)
$$

Proof of Theorem 1. The assertion follows directly from Lemmas 2 and 3.

\section{DECOMPOSITION}

In this section we will prove the main theorem, namely, that a $k$ th Peano derivative belongs to $\left[\Delta^{\prime}\right]$.

Definition 3. Suppose that a function $f$ has a $k$ th Peano derivative at every point of $\mathbf{R}$. Let

$$
\begin{aligned}
& H(f, M, \delta) \\
& \quad=\left\{x:\left|\frac{k-1}{2} f_{k}(x)+\sum_{j=0}^{k-1}(-1)^{k-1-j}\left(\begin{array}{c}
k-1 \\
j
\end{array}\right) j^{k} \varepsilon_{k}(x, j t)\right| \leq M \text { for }|t|<\delta\right\}
\end{aligned}
$$

where $M$ and $\delta$ are some positive constants. 
Theorem 2. $H=H(f, M, \delta)$ is closed and $f_{k-1}$ is differentiable on $H$ relative to $H$ with $\left(\left.f_{k-1}\right|_{H}\right)^{\prime}(x)=f_{k}(x)$, also $\left|f_{k}(x)\right| \leq 2 M$ for every $x \in H$.

Proof. Let $x \in \bar{H}$. Let $1>\varepsilon>0$ be given. There is $0<\eta<\delta$ such that $\left|\varepsilon_{k}(x, h)\right|<\varepsilon$ whenever $|h|<\eta$. Let $x_{n} \in H$ so that $\left|x_{n}-x\right|<\eta / k$. Then for $t=\left(x_{n}-x\right) \varepsilon^{1 / k}$ we have $|t|<\delta$ and $\left|x_{n}-x+j t\right|<\eta$, for $j=$ $0,1, \ldots, k-1$. Then the formula from Theorem 1 gives

$$
\begin{aligned}
& \left|\frac{f_{k-1}\left(x_{n}\right)-f_{k-1}(x)}{x_{n}-x}-f_{k}(x)\right| \\
& \leq \varepsilon^{1 / k} \frac{k-1}{2}\left|f_{k}(x)\right|+\sum_{j=0}^{k-1}\left(\begin{array}{c}
k-1 \\
j
\end{array}\right) \frac{\left(1+j \varepsilon^{1 / k}\right)^{k}}{\varepsilon^{(k-1) / k}}\left|\varepsilon_{k}\left(x, x_{n}-x+j t\right)\right| \\
& \quad+\varepsilon^{1 / k}\left|\frac{k-1}{2} f_{k}\left(x_{n}\right)+\sum_{j=0}^{k-1}(-1)^{k-1-j}\left(\begin{array}{c}
k-1 \\
j
\end{array}\right) j^{k} \varepsilon_{k}\left(x_{n}, j t\right)\right| \\
& \leq \varepsilon^{1 / k} \frac{k-1}{2}\left|f_{k}(x)\right|+\sum_{j=0}^{k-1}\left(\begin{array}{c}
k-1 \\
j
\end{array}\right)\left(1+j \varepsilon^{1 / k}\right)^{k} \varepsilon^{1 / k}+\varepsilon^{1 / k} M .
\end{aligned}
$$

Therefore as $x_{n} \rightarrow x$ with $x_{n} \in H$ we get

$$
\frac{f_{k-1}\left(x_{n}\right)-f_{k-1}(x)}{x_{n}-x}-f_{k}(x) \rightarrow 0 \text {. }
$$

Now let $x \in \bar{H},\left\{x_{n}\right\}$ a sequence in $H$ with $x_{n} \rightarrow x$, and $0 \neq|t|<\delta$. Then the formula from Theorem 1 yields

$$
\begin{aligned}
& \left|t\left(\frac{k-1}{2} f_{k}(x)+\sum_{j=0}^{k-1}(-1)^{k-1-j}\left(\begin{array}{c}
k-1 \\
j
\end{array}\right) \frac{\left(x_{n}-x+j t\right)^{k}}{t^{k}} \varepsilon_{k}\left(x, x_{n}-x+j t\right)\right)\right| \\
& \leq\left|t\left(\frac{k-1}{2} f_{k}\left(x_{n}\right)+\sum_{j=0}^{k-1}(-1)^{k-1-j}\left(\begin{array}{c}
k-1 \\
j
\end{array}\right) j^{k} \varepsilon_{k}\left(x_{n}, j t\right)\right)\right| \\
& \quad+\left|f_{k-1}\left(x_{n}\right)-f_{k-1}(x)-\left(x_{n}-x\right) f_{k}(x)\right| \\
& \leq|t| M+\left|f_{k-1}\left(x_{n}\right)-f_{k-1}(x)-\left(x_{n}-x\right) f_{k}(x)\right| .
\end{aligned}
$$

Letting $n \rightarrow \infty$ the left-hand side becomes

$$
\left|t\left(\frac{k-1}{2} f_{k}(x)+\sum_{j=0}^{k-1}(-1)^{k-1-j}\left(\begin{array}{c}
k-1 \\
j
\end{array}\right) j^{k} \varepsilon_{k}(x, j t)\right)\right|
$$

while the right-hand side is $|t| M$. Hence $x \in H$.

That $\left|f_{k}(x)\right| \leq 2 M$ on $H$ follows from the definition of $H$ taking $t=0$.

Lemma 4. $\bigcup_{M=1}^{\infty} H(f, M, 1)=\mathbf{R}$.

Proof. The assertion follows from the fact that $\varepsilon_{k}(x, j t)$ is a continuous function of $t$ for $j=0,1, \ldots, k-1$. 
The following corollary is an immediate consequence of Lemma 4 and Theorem 2.

Corollary 1 . Let $f$ be a continuous function on $\mathbf{R}$ such that $f_{k}(x)$ exists at every point of $\mathbf{R}$. Then $f_{k} \in\left[\Delta^{\prime}\right]$.

Proof. The corollary follows directly from Theorem 2, Lemma 4, and the fact that for any function $g$ defined on a closed set $P$, that is differentiable with respect to $P$, there is a function $G$ differentiable on $\mathbf{R}$ so that $\left.G\right|_{P}=g$ and $\left.G^{\prime}\right|_{P}=g^{\prime}$. (See Mařik [7].)

Definition 4. Let $f$ be a function defined on $\mathbf{R}$. If there exist a function $g$ and closed sets $A_{n}, n=1,2, \ldots$, such that $\bigcup_{n=1}^{\infty} A_{n}=\mathbf{R}$ and $\left.g\right|_{A_{n}} ^{\prime}(x)=f(x)$ for $x \in A_{n}$, then we say that $f$ is a composite derivative of $g$.

Corollary 2. $f_{k}$ is a composite derivative of $f_{k-1}$.

An immediate consequence of this result is the following corollary, first proved by Zygmund and Marcinkiewicz. (See Zygmund [12, p. 77].)

Corollary 3. $f_{k}$ is the approximate derivative of $f_{k-1}$ almost everywhere.

\section{ON $(k-1)$ th Peano derivatives}

It was known that for any point $x$ there is a sequence $x_{n} \rightarrow x$ so that

$$
\lim _{n \rightarrow \infty}\left(f_{k-1}\left(x_{n}\right)-f_{k-1}(x)\right) /\left(x_{n}-x\right)=f_{k}(x) .
$$

(See Weil [11] or Mařik [6].) In this section we will prove that if $f_{k}$ exists at some point $x$ and $f_{k-1}$ exists at some neighborhood of the point $x$, then there is a perfect set $P$ of positive measure such that $x$ is a bilateral point of accumulation of $P$ and $f_{k-1}$ differentiates at $x$ along $P$ with $\left.f_{k-1}\right|_{P} ^{\prime}(x)=$ $f_{k}(x)$. In order to prove the above we need a few lemmas, two of which (Lemma 5 and Lemma 7) are known. (See Corominas [3].)

Lemma 5. Let $f$ and $g$ be functions on $\mathbf{R}$ such that the nth Peano derivatives $f_{n}(x)$ and $g_{n}(x)$ exist at some point $x$. Then the function $f g$ has an nth Peano derivative at $x$ and

$$
(f g)_{n}(x)=\sum_{j=0}^{n}\left(\begin{array}{l}
n \\
j
\end{array}\right) f_{j}(x) g_{n-j}(x) .
$$

Lemma 6. Let $f$ and $g$ be functions on $\mathbf{R}$ such that the nth Peano derivative, $f_{n}(x)$, and the nth ordinary derivative, $g^{(n)}(x)$, exist at some point $x$. Then

$$
\sum_{j=0}^{n}(-1)^{j}\left(\begin{array}{l}
n \\
j
\end{array}\right)\left(f g^{(j)}\right)_{n-j}(x)=f_{n}(x) g(x) .
$$


Proof. By Lemma 5

$$
\begin{aligned}
& \sum_{j=0}^{n}(-1)^{j}\left(\begin{array}{l}
n \\
j
\end{array}\right)\left(f g^{(j)}\right)_{n-j}(x) \\
& =\sum_{j=0}^{n}(-1)^{j}\left(\begin{array}{l}
n \\
j
\end{array}\right) \sum_{i=0}^{n-j}\left(\begin{array}{c}
n-j \\
i
\end{array}\right) f_{i}(x)\left(g^{(j)}\right)_{(n-j-i)}(x) \\
& =\sum_{j=0}^{n}(-1)^{j}\left(\begin{array}{c}
n \\
j
\end{array}\right) \sum_{i=0}^{n-j}\left(\begin{array}{c}
n-j \\
i
\end{array}\right) f_{i}(x) g^{(n-i)}(x) \\
& =\sum_{i=0}^{n} \sum_{j=0}^{n-i}(-1)^{j}\left(\begin{array}{c}
n \\
j
\end{array}\right)\left(\begin{array}{c}
n-j \\
i
\end{array}\right) f_{i}(x) g^{(n-i)}(x) \\
& =\sum_{i=0}^{n} \sum_{j=0}^{n-i}(-1)^{j}\left(\begin{array}{c}
n-i \\
j
\end{array}\right)\left(\begin{array}{c}
n \\
i
\end{array}\right) f_{i}(x) g^{(n-i)}(x) \\
& =\sum_{i=0}^{n}\left(\begin{array}{c}
n \\
i
\end{array}\right) \sum_{j=0}^{n-i}(-1)^{j}\left(\begin{array}{c}
n-i \\
j
\end{array}\right) f_{i}(x) g^{(n-i)}(x) \\
& =f_{n}(x) g(x)+\sum_{i=0}^{n-1}\left(\begin{array}{c}
n \\
i
\end{array}\right)(1-1)^{n-i} f_{i}(x) g^{(n-i)}(x)=f_{n}(x) g(x) \text {. }
\end{aligned}
$$

Lemma 7. Let $H$ be a function defined in a neighborhood $\mathscr{O}$ of a point $y$. Suppose that $H$ is $n$ times Peano differentiable in $\odot$ and that $H_{n}$ is $m$ times Peano differentiable in $\mathcal{O}$. Then $H$ is $(n+m)$ times Peano differentiable at $y$, and $H_{(n+m)}(y)=\left(H_{n}\right)_{m}(y)$.

Lemma 8. Let $f$ be defined in some neighborhood $\mathscr{O}$ of 0 . Suppose that the $k$ th Peano derivative of $f$ at 0 exists and that the lth Peano derivative of $f$ exists in $\mathcal{O}$, where $k$ and $l$ are positive integers with $l \leq k-1$. Also suppose that $f(0)=f_{1}(0)=\cdots=f_{k}(0)=0$. Let $g(y)=y^{-(k-l)}$. Then the function $h_{i}$ defined by

$$
\begin{aligned}
h(y)= & \left(\begin{array}{l}
l \\
0
\end{array}\right) f(y) g(y)-\left(\begin{array}{l}
l \\
1
\end{array}\right) \int_{0}^{y} f(t) g^{\prime}(t) d t \\
& +\cdots+(-1)^{l}\left(\begin{array}{l}
l \\
l
\end{array}\right) \int_{0}^{y} \int_{0}^{x_{2}} \cdots \int_{0}^{x_{l-1}} f(t) g^{(l)}(t) d t \cdots d x_{2} \quad \text { for } y \neq 0,
\end{aligned}
$$

and $h(0)=0$ has an lth Peano derivative on $\mathscr{O}$.

Moreover,

$$
h_{l}(y)= \begin{cases}f_{l}(y) / y^{k-l} & \text { if } y \neq 0, \\ 0 & \text { if } y=0 .\end{cases}
$$

Proof. By assumption, $f(y)=y^{k} \varepsilon_{k}(0, y)$. Consequently all of the above integrals are integrals of continuous functions. Hence $h$ is well defined. Moreover, 
for $y \neq 0, y \in \mathscr{O}$

$$
H(y)=\int_{0}^{y} \int_{0}^{x_{2}} \cdots \int_{0}^{x_{i-1}} f(t) g^{(i)}(t) d t \cdots d x_{2}, \quad i=1, \ldots, l,
$$

is $i$ times ordinarily differentiable and $H^{(i)}(y)=f(y) g^{(i)}(y)$ for $i=1, \ldots, l$. By Lemma $5, f g^{(i)}$ is $l$ times Peano differentiable at $y$. Therefore by Lemma $7, H$ is $l$ times Peano differentiable at $y$ and

$$
H_{l}(y)=\left(H^{(i)}\right)_{l-i}(y)=\left(f(y) g^{(i)}(y)\right)_{(l-i)} .
$$

Hence $h$ is $l$ times Peano differentiable at $y$ and

$$
h_{l}(y)=\sum_{j=0}^{l}(-1)^{j}\left(\begin{array}{l}
l \\
j
\end{array}\right)\left(f g^{(j)}\right)_{(l-j)}(y),
$$

and, by Lemma 6, $h_{l}(y)=f_{l}(y) g(y)$.

It remains to prove that $h_{l}(0)$ exists and that $h_{l}(0)=0$. For $y \neq 0$

$$
\begin{aligned}
\frac{h(y)}{y^{l}}=\frac{1}{y^{l}}\left\{\left(\begin{array}{l}
l \\
0
\end{array}\right) y^{l} \varepsilon_{k}(0, y)+(k-l)\left(\begin{array}{l}
l \\
1
\end{array}\right) \int_{0}^{y} t^{l-1} \varepsilon_{k}(0, t) d t\right. \\
+\cdots+(k-l)(k-l+1) \cdots(k-1) \\
\left.\cdot\left(\begin{array}{l}
l \\
l
\end{array}\right) \int_{0}^{y} \int_{0}^{x_{2}} \cdots \int_{0}^{x_{l-1}} \varepsilon_{k}(0, t) d t \cdots d x_{2}\right\} .
\end{aligned}
$$

Hence $\lim _{y \rightarrow 0}\left(h(y) / y^{l}\right)=0$. Therefore $h(0)=h_{1}(0)=\cdots=h_{l}(0)=0$.

Now suppose that $f$ has an $l$ th Peano derivative in some neighborhood $\mathscr{O}$ of a point $x$ and that $f_{k}(x)$ exists. Consider a function $T(y)=f(y)-f(x)-$ $(y-x) f_{1}(x)-\cdots-(y-x)^{k} f_{k}(x) / k !$ and its translate $G(t)=T(x+t)$.

Then $G$ satisfies the hypothesis of Lemma 8 and by that lemma the function $H$ defined by

$$
\begin{aligned}
H(y)= & \left(\begin{array}{l}
l \\
0
\end{array}\right) G(y) g(y)-\left(\begin{array}{l}
l \\
1
\end{array}\right) \int_{0}^{y} G(t) g^{\prime}(t) d t \\
& +\cdots+(-1)^{l}\left(\begin{array}{l}
l \\
l
\end{array}\right) \int_{0}^{y} \int_{0}^{x_{2}} \cdots \int_{0}^{x_{l-1}} G(t) g^{(l)}(t) d t \cdots d x_{2} \text { for } y \neq 0
\end{aligned}
$$

and $H(0)=0$ has an $l$ th Peano derivative on $x-\mathscr{O}$.

Moreover, by the same lemma,

$$
H_{l}(y)= \begin{cases}G_{l}(y) / y^{k-l} & \text { if } y \neq 0, \\ 0 & \text { if } y=0 .\end{cases}
$$

But

$$
G_{l}(t)=T_{l}(t+x)=f_{l}(t+x)-f_{l}(x)-t f_{l+1}(x)-\cdots-t^{k-l} \frac{f_{k}(x)}{(k-l) !} .
$$

Therefore we have proved the following theorem.

Theorem 3. Suppose that a function $f$ in some neighborhood $\theta$ of a point $x$ has an lth Peano derivative in $\mathscr{\theta}$ and $a$ kth Peano derivative at $x$, where 
$0 \leq l \leq k$. Then the function $F$ defined on $\mathscr{O}$ by

$$
F(y)= \begin{cases}\left(f_{l}(y)-\sum_{j=0}^{k-l}(y-x)^{j}\left(f_{l+j}(x)\right) / j !\right) /(y-x)^{k-l} & \text { if } y \neq x, \\ 0 & \text { if } y=x\end{cases}
$$

is an lth Peano derivative.

Corollary 4. Suppose that a function $f$ defined in some neighborhood $\mathscr{O}$ of a point $x$ has a $(k-1)$ th Peano derivative in $\mathscr{O}$ and $k$ th Peano derivative at $x$. Then there exists a perfect set $P \subset \mathscr{O}$ of positive measure such that $x$ is a bilateral point of accumulation of $P$ and

$$
\lim _{y \in P, y \rightarrow x} \frac{f_{k-1}(y)-f_{k-1}(x)}{y-x}=f_{k}(x) .
$$

Proof. The function $F$ from Theorem 3, applied with $l=k-1$ is a $(k-1)$ th Peano derivative and hence Baire 1, Darboux, and has Denjoy property. Therefore, there is a perfect set $P$ of positive measure such that $x$ is a bilateral point of accumulation of $P$ and such that $F$ is continuous at $x$ with respect to $P$.

\section{6. $A_{n}$ CAN BE CHOSEN TO BE PERFECT}

In this section we will prove that the sets $A_{n}$ from the definition of $\left[\Delta^{\prime}\right]$ for Peano derivatives can be chosen to be perfect.

Let $y \in H(f, M, 1)$ be an isolated point of $H(f, M, 1)$. Then there is a $1>\delta(y)>0$ so that $(y-2 \delta(y), y+2 \delta(y)) \cap H(f, M, 1)=\{y\}$. Let $P_{y}$ be a perfect set containing $y$ so that $y$ is a bilateral point of accumulation of $P_{y}$ satisfying

$$
\lim _{z \in P_{y}, z \rightarrow y} \frac{f_{k-1}(z)-f_{k-1}(y)}{z-y}=f_{k}(y)
$$

and

$$
\left|\frac{f_{k-1}(z)-f_{k-1}(y)}{z-y}-f_{k}(y)\right| \leq 1 \text { for every } z \in P_{y} .
$$

Corollary 4 assures the existence of $P_{y}$. If $P_{y} \cap\left(y+\frac{1}{n+1}, y+\frac{1}{n}\right) \neq \varnothing$, for $n \in \mathbf{Z} \backslash\{-1,0\}$, then by the Baire category theorem there is $Q_{n}(y) \subset P_{y} \cap$ $\left(y+\frac{1}{n+1}, y+\frac{1}{n}\right)$, such that $Q_{n}$ is perfect and that there is $M_{n} \in \mathbf{N}$ with $Q_{n}(y) \subset H\left(f, M_{n}, 1\right)$. Let

$$
Q_{y}=\bigcup_{n \in \mathbf{Z} \backslash\{-1,0\}} Q_{n}(y) \cap\left(y-\delta^{2}(y), y+\delta^{2}(y)\right) \cup\{y\},
$$

and let

$$
H_{M}=H(f, M, 1) \cup\left\{Q_{y}: y \in H(f, M, 1), \quad y \text { is isolated in } H(f, M, 1)\right\} \text {. }
$$

Theorem 4. $H_{M}$ is a perfect set, and $f_{k-1}$ is differentiable on $H_{M}$ relative to $H_{M}$ with $\left(\left.f_{k-1}\right|_{H_{M}}\right)^{\prime}(x)=f_{k}(x)$.

Proof. By the construction of $H_{M}$ we see that no point is an isolated point. Note that each of $Q_{y}$ is perfect and that $Q_{y} \cap Q_{z}=\varnothing$ if $y, z \in H(f, M, 1)$ are two different isolated points of $H(f, M, 1)$. Suppose that $H_{M}$ is not closed. Then there is a sequence $\left\{z_{n}\right\}$ and a point $z$ such that $\lim _{n \rightarrow \infty} z_{n}=z$ 
and $\left\{z_{n}\right\} \cap H(f, M, 1)=\varnothing$, but then either there is a subsequence $\left\{z_{n_{k}}\right\} \subset$ $\left\{z_{n}\right\}$ and $y \in H(f, M, 1)$ with $y$ an isolated point of $H(f, M, 1)$ so that $\left\{z_{n_{k}}\right\} \subset Q_{y}$, or there is a sequence $\left\{y_{n_{k}}\right\} \subset H(f, M, 1)$ so that $y_{n_{k}}$ is an isolated point of $H(f, M, 1)$ and $z_{n_{k}} \in Q_{y_{n_{k}}}$ for $k=1,2, \ldots$ In the first case $z \in Q_{y} \subset H_{M}$, and in the second $y_{n_{k}} \rightarrow z$ and hence $z \in H(f, M, 1)$.

Now if $x \in H_{M}$ is an isolated point of $H(f, M, 1)$, then clearly $f_{k-1}^{\prime}$ at $x$ relative to $H_{M}$ exists and is equal to $f_{k}(x)$. If $x \in Q_{y}$ for some $y \in$ $H(f, M, 1)$ where $y$ is an isolated point of $H(f, M, 1)$, then there is $n \in$ $\mathbf{Z}$ so that $x \in Q_{n}(y) \subset H\left(f, M_{n}(y), 1\right)$, and by the fact that there are two numbers $a<b$ so that $(a, b) \cap H_{M}=Q_{n}(y)$, we see that $f_{k-1}^{\prime}$ at $x$ relative to $H_{M}$ exists and is equal to $f_{k}(x)$.

Finally let $x \in H(f, M, 1)$, and $x$ not an isolated point of $H(f, M, 1)$. Let $\varepsilon>0$ be given. Then there is $\varepsilon>\eta>0$ so that

$$
\left|\frac{f_{k-1}(y)-f_{k-1}(x)}{y-x}-f_{k}(x)\right|<\varepsilon
$$

whenever $y \in H(f, M, 1)$ and $|y-x|<\eta$.

Let $y$ be an isolated point of $H(f, M, 1)$, and let $z \in Q_{y}$ with $|z-x|<$ $\eta / 2$. Since $|y-z|<\delta^{2}(y)<\delta(y)$ and $|y-x|>2 \delta(y)$, we have $\eta / 2>$ $|x-z| \geq|x-y|-|y-z|>2 \delta(y)-\delta(y)=\delta(y)$. Hence $|y-x| \leq|y-z|+$ $|z-x|<\delta(y)+\eta / 2<\eta$.

Now

$$
\begin{aligned}
\left|\frac{f_{k-1}(z)-f_{k-1}(x)}{z-x}-f_{k}(x)\right| \\
=\mid\left(\frac{f_{k-1}(y)-f_{k-1}(x)}{y-x}-f_{k}(x)\right) \frac{y-x}{z-x} \\
\quad \quad+\left(\frac{f_{k-1}(z)-f_{k-1}(y)}{z-y}-f_{k}(y)\right) \frac{z-y}{z-x}+\frac{z-y}{z-x}\left(f_{k}(y)-f_{k}(x)\right) \mid \\
\leq\left|\frac{f_{k-1}(y)-f_{k-1}(x)}{y-x}-f_{k}(x)\right|\left|1-\frac{z-y}{z-x}\right| \\
\quad+\left|\frac{f_{k-1}(z)-f_{k-1}(y)}{z-y}-f_{k}(y)\right|\left|\frac{z-y}{z-x}\right|+\left|\frac{z-y}{z-x}\right|\left(\left|f_{k}(x)\right|+\left|f_{k}(y)\right|\right) \\
\leq \varepsilon\left(1+\frac{\delta^{2}(y)}{\delta(y)}\right)+1 \cdot \frac{\delta^{2}(y)}{\delta(y)}+\frac{\delta^{2}(y)}{\delta(y)} 4 M \\
\leq 2 \varepsilon+\delta(y)(1+4 M) \leq 2 \varepsilon+\frac{\varepsilon}{2}(1+4 M),
\end{aligned}
$$

and since $\varepsilon$ was arbitrary, we have that $f_{k-1}^{\prime}$ at $x$ relative to $H_{M}$ exists and equals $f_{k}(x)$.

\section{ACKNOWLEDGMENTS}

The author would to thank Professor C. E. Weil, his thesis advisor, for the generous help he offered during the preparation of this paper. 


\section{REFERENCES}

1. S. Agronsky, R. Biskner, A. Bruckner, and J. Mařik, Representations of functions by derivatives, Trans. Amer. Math. Soc. 263 (1981), 493-500.

2. A. M. Bruckner, Differentiation of real functions, Lecture Notes in Math., vol. 659, Springer, Berlin and New York, 1978.

3. M. E. Corominas, Contribution a la théorie de la dérivation d'order supérieur, Bull. Soc. Math. France 81 (1953), 176-222.

4. H. Oliver, The exact Peano derivative, Trans. Amer. Math. Soc. 76 (1954), 444-456.

5. R. J. O’Malley, Decomposition of approximate derivatives, Proc. Amer. Math. Soc. 69 (1978), 243-247.

6. J. Mařik, On generalized derivatives, Real Anal. Exchange 3 (1977-78), 87-92.

7. _ Derivatives and closed sets, Acta Math. Hungar. 43 (1984), 25-29.

8. S. Verblunsky, On the Peano derivatives, Proc. London Math. Soc. (3) 22 (1971), 313-324.

9. C. Weil, On properties of derivatives, Trans. Amer. Math. Soc. 114 (1965), 363-376.

10. __ On approximate and Peano derivatives, Proc. Amer. Math. Soc. 20 (1969), 487-490.

11. __ A property for certain derivatives, Indiana Univ. Math. J. 23 (1973/74), 527-536.

12. A. Zygmund, Trigonometric series, 2nd ed., Cambridge Univ. Press, Cambridge, 1959.

Department of Mathematics, Michigan State University, East Lansing, Michigan 48824

Current address: Department of Mathematics, California State University, San Bernardino, California 92407

E-mail address: hajrudin@math.msu.edu 